\title{
Construção de Códigos Esféricos através do Reticulado Hexagonal
}

C. ALVES 1 , Departamento de Matemática, Instituto de Geociências e Ciências Exatas, UNESP - Universidade Estadual Paulista, 13506-900 Rio Claro, SP, Brasil

A.A. de ANDRADE2, Departamento de Matemática, Instituto de Biociências, Letras e Ciências Exatas, UNESP - Universidade Estadual Paulista, 15054-000 São José do Rio Preto, SP, Brasil

S.I.R. $\operatorname{COSTA}^{3}$, Departamento de Matemática, Instituto de Matemática, Estatística e Computação Científica, Universidade Estadual de Campinas, 13083-859 Campinas, SP, Brasil.

\begin{abstract}
Resumo. Códigos esféricos $n$-dimensionais gerados por grupos comutativos em dimensão par, $n=2 m$, podem ser determinados pelo quociente de reticulados $m$-dimensionais, quando os vetores que geram o sub-reticulado são mutuamente ortogonais [4]. Apresentamos a construção de sub-reticulados nestas condições, a partir do reticulado hexagonal, $A_{2}$. Comparamos a distância mínima do código esférico construído através do quociente destes reticulados com o limitante da distância mínima estabelecido em [5].
\end{abstract}

Palavras-chave. Reticulados, códigos esféricos, distância mínima.

\section{Introdução}

Para códigos esféricos, características como boa distância mínima, regiões de decisão simétricas e perfil de distâncias homogêneo da constelação de sinais são determinantes para uma baixa probabilidade de erro na transmissão de sinais através de um canal gaussiano.

Nesse sentido, a utilização de códigos de grupo comutativo merece destaque. Para esses códigos, a análise da distância mínima desempenha papel fundamental, visto que sua estrutura algébrica garante as características de simetria desejadas.

Em [6], Slepian estabeleceu, de maneira geral, os conceitos sobre códigos esféricos para o canal gaussiano e apresentou a teoria necessária para a construção dos códigos gerados por grupos de matrizes ortogonais que geram pontos sobre a superfície de uma hiperesfera, de modo uniforme.

\footnotetext{
${ }^{1}$ carina_matematica@yahoo.com.br

2andrade@ibilce.unesp.br

${ }^{3}$ sueli@ime.unicamp.br
} 
De maneira geral, dada uma dimensão $n$ e um número de pontos $M$ procurase por um código de grupo $[M, n]$ com a maior distância mínima. Este código é chamado ótimo. Os principais esforços para resolver este problema são:

- Construção de limitantes para o número de pontos $M=M(n, d)$ de um código esférico que envolva a dimensão $n$ e a distância mínima $d$.

- Construção de códigos que tenham distâncias mínimas próximas da distância limite.

- Determinação do melhor vetor inicial da esfera unitária do $\mathbb{R}^{n}$ que, para um determinado grupo gerador, maximiza a distância mínima entre dois pontos quaisquer do código.

De acordo com [2], o número de casos que devem ser verificados para encontrar o código ótimo é aproximadamente $\left(\begin{array}{c}M / 2 \\ n / 2\end{array}\right)$. Para grupos que geram um grande número de pontos, a busca pelo código ótimo usando técnicas de programação, torna-se um problema computacional de custo muito alto, o que motivou-nos a utilizar ferramentas que gerassem um procedimento possível para, em casos especiais, gerar códigos de grupos comutativos muito bons, onde para calcular a distância mínima calcula-se a distância entre as imagens dos vetores de norma mínima da base que gera o reticulado e o vetor inicial, sem a necessidade de analisar casos.

Códigos esféricos em dimensão par, gerados por grupos comutativos de matrizes ortogonais, podem ser determinados pelo quociente de dois reticulados na metade da dimensão quando o sub-reticulado é "retangular" (isto é, quando os vetores que o geram são mutuamente ortogonais), [2] e [4]. Assim, o objetivo deste trabalho é a construção de códigos esféricos através do quociente de reticulados, com a finalidade de obter códigos esféricos em que a distância mínima se aproxime do limitante da distância mínima estabelecido em [5].

$\mathrm{Na}$ bibliografia pesquisada, verifica-se a existência de sub-reticulados "retangulares" a partir do reticulado hexagonal $A_{2}$, que é o de maior densidade de empacotamento conhecida em dimensão 2 e compara-se a distância mínima do código esférico construído através do quociente destes reticulados com o limitante.

Este trabalho é organizado como segue. Na Seção 2, apresentamos algumas definições e resultados usados para a construção de códigos esféricos a partir do quociente de reticulados; na Seção 3, descrevemos a expressão para o cálculo da distância mínima; na Seção 4, exibimos o limitante para códigos de grupo comutativo; na Seção 5, apresentamos um resultado que caracteriza a forma geral dos sub-reticulados de $A_{2}$ que satisfazem a condição de ortogonalidade exigida juntamente com um exemplo onde a distância mínima encontrada é comparada com o limitante. Finalmente, na Seção 6, conclusões são dadas.

\section{Reticulados e Códigos de Grupo}

Definição 2.1 ([1]- Reticulado). Um reticulado $\Lambda_{\beta}$ é o conjunto de todas as combinações lineares com coeficientes inteiros de um conjunto $\beta=\left\{w_{1}, \cdots, w_{m}\right\}$ de $m$ 
vetores linearmente independentes do espaço vetorial $\mathbb{R}^{m}$,

$$
\Lambda_{\beta}=\left\{\mathbf{x}=\sum_{i=1}^{m} a_{i} w_{i} \mid a_{i} \in \mathbb{Z}, \forall i\right\} .
$$

$O$ conjunto $\beta$ é denominado uma base de $\Lambda_{\beta}$.

O reticulado $A_{n}=\left\{\left(x_{1}, \ldots, x_{n+1}\right) \in \mathbb{Z}^{n+1} \mid x_{1}+\cdots+x_{n+1}=0\right\}$ é chamado de reticulado hexagonal para $n=2$. Sua matriz geradora é $\left\{(1,0),\left(\frac{1}{2}, \frac{\sqrt{3}}{2}\right)\right\}$.

Definição 2.2 ([1] - Sub-reticulado). Seja $B$ uma matriz $n \times n$ com entradas inteiras. Um sub-reticulado de $\Lambda_{\beta}$ é dado por $\Lambda_{\alpha}=\left\{\mathbf{x}=\lambda B M \mid \lambda \in \mathbb{Z}^{n}\right.$, onde $M$ é a matriz geradora do reticulado $\Lambda_{\beta}$.

O índice do sub-reticulado $\Lambda_{\alpha}$ é a cardinalidade do grupo quociente $\Lambda_{\beta} / \Lambda_{\alpha}$ e

$$
\left|\Lambda_{\beta} / \Lambda_{\alpha}\right|=\frac{\operatorname{vol}\left(\Lambda_{\alpha}\right)}{\operatorname{vol}\left(\Lambda_{\beta}\right)}=\frac{\sqrt{\operatorname{det}\left(\Lambda_{\alpha}\right)}}{\sqrt{\operatorname{det}\left(\Lambda_{\beta}\right)}}=|\operatorname{det}(B)| .
$$

Dado um empacotamento no $\mathbb{R}^{n}$, associado ao reticulado $\Lambda_{\beta}$, definimos a sua densidade de empacotamento de esferas de raio $r$ como sendo a proporção do espaço $\mathbb{R}^{n}$ coberta pela união das esferas.

Um código esférico é um subconjunto finito da esfera unitária euclidiana $S^{n}$, contida em $\mathbb{R}^{n+1}$. A distândia mínima de um código esférico $n$-dimensional $\mathcal{C} \subset S^{n}$ é definida como

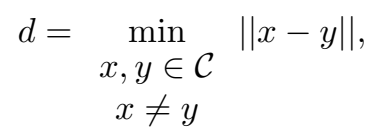

onde $\|x-y\|$ é a distância euclidiana em $\mathbb{R}^{n+1}$ entre os pontos do código $x$ e $y$.

Definição 2.3 ([1]- Órbita). Seja $x_{0} \in \mathbb{R}^{n}$ e G um grupo de matrizes $n \times n$. Chamamos de órbita de $x_{0}$ por $G$ ao conjunto

$$
G\left(x_{0}\right)=\left\{g\left(x_{0}\right) \mid g \in G\right\} .
$$

Definição 2.4 ([1]- Código de Grupo). Um código de grupo $\mathcal{C}$ é a órbita de um vetor $v$ na esfera unitária $S^{n-1}$ por um subgrupo $G=\left\{O_{i}\right\}_{i=1}^{M}$ do grupo das matrizes ortogonais $n \times n, \mathbf{O}(n)$, tal que o código $\mathcal{C}=\left\{O_{i} v\right\}_{i=1}^{M}$ é substancial em $\mathbb{R}^{n}$ (não está contido em um hiperespaço, isto é, subespaço vetorial de codimensão 1).

Quando o subgrupo de $\mathbf{O}(n)$ for comutativo, teremos um código de grupo comutativo.

A alocação de pontos em uma hiperesfera com a maior distância euclidiana mínima depende da estrutura do grupo que é admitido no processo.

Teorema 2.1 ([1]). Sejam $\alpha=\left\{v_{1}, \cdots, v_{m}\right\}$ e $\beta=\left\{w_{1}, \cdots, w_{m}\right\}$ duas bases de $\mathbb{R}^{m}$, $\Lambda_{\alpha}$ e $\Lambda_{\beta}$ os reticulados gerados por $\alpha$ e $\beta$, respectivamente, e $\Lambda_{\alpha} \subset \Lambda_{\beta}$. Se $A=\left(a_{i j}\right)$, $a_{i, j} \in \mathbb{Z}$ é a matriz da base $\alpha$ escrita em relação à base $\beta$, então a classificação $e$ o conjunto de geradores do grupo $\Lambda_{\beta} / \Lambda_{\alpha}$ são obtidos da forma normal de Smith de A. 
Definição 2.5 ([1]- Forma Normal de Smith). Dizemos que uma matriz $A=\left(a_{i, j}\right)$ de ordem $n \times n$ esta na forma normal de Smith se A é uma matriz diagonal com coeficientes inteiros não negativos tal que $a_{i, i} \mid a_{i+1, i+1}$ para todo $i<n$.

\section{Expressão para a Distância na Imagem por $\psi$}

Seja

$$
\begin{aligned}
\psi: & \mathbb{R}^{m} \longrightarrow \mathbb{R}^{2 m} \\
& y \longmapsto \psi(y)=\left(\delta_{1} \cos \left(\frac{y_{1}}{\delta_{1}}\right), \delta_{1} \operatorname{sen}\left(\frac{y_{1}}{\delta_{1}}\right), \cdots, \delta_{m} \cos \left(\frac{y_{m}}{\delta_{m}}\right), \delta_{m} \operatorname{sen}\left(\frac{y_{m}}{\delta_{m}}\right)\right)(3.1
\end{aligned}
$$

onde $y=\left(y_{1}, \cdots, y_{m}\right)$ são coordenadas em relação a uma base ortogonal.

A parametrização $\psi$ induz uma relação de equivalência em $\mathbb{R}^{m}$ cujas classes formam um conjunto chamado toro planar abstrato. Um conjunto de representantes para essa relação é o paralelepípedo $\prod_{i=1}^{m}\left[0,2 \pi \delta_{i}\right)$. Este conjunto de representantes pode ser visto como um espaço quociente onde os lados paralelos do paralelepípedo são identificados.

Assim, considerando um toro $T_{\delta=\left(\delta_{1}, \cdots, \delta_{m}\right)}$ na esfera unitária contida em $\mathbb{R}^{2 m} \mathrm{e}$ a aplicação (3.1), com $\delta_{i}=\frac{\left\|v_{i}\right\|}{2 \pi}$, definimos a distância euclidiana ao quadrado entre $\psi(x)$ e $\psi(y)$ na esfera de $\mathbb{R}^{2 m}$ por

$$
\begin{aligned}
d^{2}(\psi(x), \psi(y)) & =\|\psi(x)-\psi(y)\|^{2} \\
& =4 \sum_{i=1}^{m}\left(\frac{\left\|v_{i}\right\|}{\left\|v_{1}\right\|+\cdots+\left\|v_{m}\right\|}\right)^{2} \operatorname{sen}^{2}\left(\frac{\pi\left(x_{i}-y_{i}\right)}{\left\|v_{i}\right\|}\right) \\
& =4 \sum_{i=1}^{m} \delta_{i}^{2} \operatorname{sen}^{2}\left(\frac{x_{i}-y_{i}}{2 \delta_{i}}\right) .
\end{aligned}
$$

Um caso particular que utilizaremos no caso de grupos comutativos é $y=\mathbf{0}=$ $(0, \cdots, 0)$, pois sendo estes geometricamente uniformes, o perfil de distâncias a um ponto é igual para todos os pontos, portanto podemos escolher $\psi(\mathbf{0})$ para estes cálculos.

$$
\begin{aligned}
d^{2}(\psi(x), \psi(\mathbf{0})) & =\|\psi(x)-\psi(\mathbf{0})\|^{2} \\
& =4 \sum_{i=1}^{m}\left(\frac{\left\|v_{i}\right\|}{\left\|v_{1}\right\|+\cdots+\left\|v_{m}\right\|}\right)^{2} \operatorname{sen}^{2}\left(\frac{\pi x_{i}}{\left\|v_{i}\right\|}\right) .
\end{aligned}
$$

\section{Limitantes para Códigos de Grupo Comutativo}

Consideremos um código em $T_{\delta}$ com $M$ pontos e distância mínima $d$. Isto equivale a um empacotamento de $M$ chapéus esféricos sobre $T_{\delta}$ de maneira que seus centros distem entre si no mínimo $d$. Como a área $\frac{n}{2}$-dimensional ocupada por estes chapéus é no máximo a área do próprio toro $T_{\delta}$, o número de chapéus também é limitado. Vamos apresentar um limitante para $M$ estabelecido em [5], supondo que a distância mínima $d$ é fixa. 
Definição 4.1 ([1]- Chapéu esférico). Um chapéu esférico sobre o toro $T_{\delta}$ centrado em $x_{0}$ e de raio $\rho=\frac{d}{2}$ é definido por

$$
B^{T_{\delta}}\left(x_{0}, \rho\right)=\left\{x \in T_{\delta} ;\left\langle x_{0}-x, x_{0}-x\right\rangle^{1 / 2} \leq \rho\right\} .
$$

Proposição 4.1 ([5]). Todo código de grupo comutativo $G(u)=\{O(u), O \in G\}, u \in$ $S^{n-1}$ de ordem $M$ em $\mathbb{R}^{2 m}$ livre de blocos de reflexão $2 \times 2$ com distância mínima $d$ e vetor inicial $u=\left(u_{1}, \cdots, u_{2 m}\right)$ satisfaz

$$
d \leq 4 \operatorname{sen}\left(\pi\left(\frac{m^{-1 / 2} \cdot \Lambda_{m}^{1 / m}}{M^{1 / m}}\right)\right)
$$

onde $\Lambda_{m}$ é a densidade de centro máxima de um reticulado em $\mathbb{R}^{m}$.

Para $M$ grande, $d$ será pequeno e a imagem inversa do chapéu esférico estará arbitrariamente mais próxima do empacotamento reticulado em $\mathbb{R}^{m}$ e portanto estarão próximos do limitante estabelecido aqui.

Para fins comparativos, este resultado será de grande importância pois construímos códigos esféricos com o objetivo de obter distâncias mínimas mais próximas do limitante da Proposição 4.1.

\section{Construção de Sub-reticulados}

Considere o reticulado $\Lambda_{\beta}=A_{2}, \operatorname{com} \beta=\left\{w_{1}=(1,0), w_{2}=\left(\frac{1}{2}, \frac{\sqrt{3}}{2}\right)\right\}$, uma base de $A_{2}$. Nosso interesse é encontrar um sub-reticulado $\Lambda_{\alpha}$ de $\Lambda_{\beta}$, tal que $\alpha=\left\{v_{1}, v_{2}\right\}$ seja uma base ortogonal de $\Lambda_{\beta}$, isto é, $\left\langle v_{1}, v_{2}\right\rangle=0$.

Teorema 5.1 ([1]). Os sub-reticulados de $A_{2}$ que admitem uma base $\alpha=\left\{v_{1}, v_{2}\right\}$ ortogonal são da forma $\Lambda_{\alpha}=<v_{1}, v_{2}>$, com

$$
v_{1}=l_{1} v_{1}^{*} \text { e } v_{2}=l_{2} v_{2}^{*}, \quad l_{1}, l_{2} \in \mathbb{Z}^{*},
$$

onde $v_{1}^{*}$ e $v_{2}^{*}$ podem ser das formas

i) $v_{1}^{*}=a w_{1}+b w_{2}, \operatorname{com} \operatorname{mdc}(a, b)=1 e$

$$
\left\{\begin{array}{l}
v_{2}^{*}=-(a+2 b) w_{1}+(2 a+b) w_{2}, \\
\text { ou } \\
v_{2}^{*}=-\frac{(a+2 b)}{3} w_{1}+\frac{(2 a+b)}{3} w_{2}, \text { caso } 3 \mid(-(a+2 b)) \text { e } 3 \mid(2 a+b) .
\end{array}\right.
$$

ii) $v_{1}^{*}=w_{1}$ e $v_{2}^{*}=-w_{1}+2 w_{2}$

iii) $v_{1}^{*}=w_{2}$ e $v_{2}^{*}=-2 w_{1}+w_{2}$

Chamaremos de geradores primitivos os vetores $v_{1}^{*}$ e $v_{2}^{*}$ do teorema acima. Aqui consideramos os sub-reticulados do tipo (i) do Teorema (5.1), com

$$
v_{1}^{*}=a w_{1}+b w_{2} \text { e } v_{2}^{*}=-(a+2 b) w_{1}+(2 a+b) w_{2} .
$$


Estes sub-reticulados apresentam melhor desempenho quando mergulhados nos toros planares de esferas no $\mathbb{R}^{4}$ quando satisfazem:

1. $\left\|v_{1}^{*}\right\| \approx\left\|v_{2}^{*}\right\|$, ou seja, a "caixa" que define o toro está mais próxima de ser "quadrada".

Os bons tamanhos ocorrerão quando tomarmos $v_{1}=l_{1} v_{1}^{*}$ e $v_{2}=l_{2} v_{2}^{*}$ com $\frac{\left|l_{1}\right|}{\left|l_{2}\right|} \approx \sqrt{3} \approx 1,73205$. Ou seja, o sub-reticulado estará mais próximo de ser "quadrado" se satisfizer esta condição.

2. Maior ângulo mínimo entre os vetores de $A_{2}$ e os vetores $v_{1} \mathbf{e} v_{2}$.

Considerando que a "caixa" seja "quadrada", temos que devido à simetria no eixo $x$ basta considerar $a>b>0$ e assim o ângulo entre $v_{1}$ e $w_{1}$ é menor do que $30^{\circ}$. A situação ótima ocorre quando $\theta=15^{\circ}$ (maior ângulo mínimo ângulos iguais entre $v_{1}$ e $w_{1}$ e entre $v_{2}$ e $\left.w_{2}\right)$ e isto ocorre quando $\frac{a}{b}=1+\sqrt{3}$.

Exemplo 5.1. Considere $a=273, b=100$. Logo, $c=-(a+2 b)=-473 e$ $d=2 a+b=646$. Observe que $\operatorname{mdc}(a, b)=1=m d c(c, d)$. Portanto, estes são geradores primitivos do reticulado $\Lambda_{\alpha}$. Assim temos,

$$
\begin{aligned}
& v_{1}^{*}=273 w_{1}+100 w_{2} \\
& v_{2}^{*}=-473 w_{1}+646 w_{2},
\end{aligned}
$$

e $M=|\operatorname{det}(A)|=\left|\operatorname{det}\left(\begin{array}{cc}273 & -473 \\ 100 & 646\end{array}\right)\right|=223658$ pontos.

Pelo Teorema (2.1), $G \simeq \mathbb{Z}_{223658}$ e o elemento $\overline{101 w_{1}+37 w_{2}}$ é um elemento de ordem 223658.

Agora, para este sub-reticulado de $A_{2}$ ter um melhor desempenho, procuramos

1. $k=\frac{a}{b} \approx 2,73205$.

2. $v_{1}=l_{1} v_{1}^{*}, v_{2}=l_{2} v_{2}^{*} \operatorname{com} \frac{\left|l_{1}\right|}{\left|l_{2}\right|}=\sqrt{3} \approx 1,73205$.

Vemos que a primeira condição é satisfeita, pois $\frac{273}{100} \approx 2,73205$. Agora, para a segunda condição tomando, por exemplo, $l_{1}=7$ e $l_{2}=4$, temos que $\frac{7}{4}=1,75 \approx \sqrt{3}$.

Assim, para que o desempenho do sub-reticulado seja bom, vamos considerar

$$
\begin{aligned}
& v_{1}=7 v_{1}^{*} \\
& v_{2}=4 v_{2}^{*} .
\end{aligned}
$$

Agora, $a=1911, b=700, c=-1892$ e $d=2584$.

Assim, $M=|\operatorname{det}(A)|=6262424$. Pelo Teorema $(2.1), G \cong \mathbb{Z}_{6262424}$ e o elemento $\overline{81 w_{1}+299 w_{2}}$ é um elemento de ordem 6262424. Portanto, $\overline{81 w_{1}+299 w_{2}}$ gera o grupo $G$. 
Escrevendo os vetores de norma minima em relação à base de $\Lambda_{\alpha}$, temos que

$$
\left\{\begin{array}{l}
w_{1}=\frac{646 \cdot v_{1}-175 \cdot v_{2}}{1565606} \\
w_{2}=\frac{1892 . v_{1}+1911 \cdot v_{2}}{6262424} \\
w_{3}=\frac{-692 . v_{1}+2611 . v_{2}}{6262424}
\end{array}\right.
$$

Logo, por (3.2),

$$
d_{\text {min }}=\min \{0.00190773,0.00190773,0.00190773\}=0.00190773 .
$$

Calculando o limitante da Proposição (4.1), obtemos que $d_{\min } \leq 0.00190778$, uma diferença de apenas 0.00000005 . Logo, este código está próximo do limitante, para 6262424 pontos. Segundo [2], seria necessário analisar aproximadamente 4902242728866 casos para encontrar o código ótimo.

Observamos que se tivéssemos calculado a distância mínima sem a exigência do ângulo e da norma obteríamos $d_{\min }=0.00939436$ e pela Proposição $(4.1) d_{\min } \leq$ 0.010095 para 223658 pontos, uma diferença de 0.000700655 .

\section{Conclusões}

A medida que o número de pontos aumenta, o número de operações envolvidas inviabiliza o cálculo, assim, para um número muito grande de pontos ou em dimensões mais altas, ainda não se sabe qual é o código ótimo.

O método que propomos neste trabalho, permite calcular diretamente um vetor inicial, distância mínima, geradores e grupo para códigos que são ótimos ou estão muito próximos destes, independente de quão grande seja o número de pontos $M$, sem a necessidade de analisar casos em uma busca exaustiva.

\footnotetext{
Abstract. Spherical codes generated by commutative group codes of orthogonal matrices in even dimensions, $2 m$, can be determined by a quotient of $m$-dimensional lattices, where the sublattice has an orthogonal basis [4]. We characterize the construction of sub-lattices in these conditions, from the hexagonal lattice, $A_{2}$ and compared the minimum distance of spherical code constructed with the limiting of the minimum distance established in [5].
}

\section{Referências}

[1] C. Alves, "Reticulados e Códigos", Tese de Doutorado, IMECC-Unicamp, 2008.

[2] E. Biglieri, M. Elia, Cyclic-group codes for the Gaussian channel, IEEE Transactions on Information Theory, 22 (1976), 624-629.

[3] H.C. Cohen, "A Course in Computational Algebraic Number Theory", SpringerVerlag, New York, 1993. 
[4] S.I.R. Costa, M. Muniz, E. Augustini, R. Palazzo, Graphs, tessellations and perfect codes on flat tori, IEEE Transactions on Information Theory, $\mathbf{5 0}$ (2004), 2363-2377.

[5] R.M. Siqueira, S.I.R. Costa, Flat tori, lattices and bounds for commutative group codes, Designs, Codes and Cryptography, 49 (2008), 307-321.

[6] D. Slepian, Group codes for the Gaussian channel, Bell Syst. Tech. Journal, 47 (1968), 575-602. 